\title{
Titel/Title: Coping: Entscheiden, wenn das kaum noch möglich ist
}

Autor*innen/Author(s): Uwe Schimank

Veröffentlichungsversion/Published version: Postprint

Zeitschriftenartikel/Journal article

\section{Empfohlene Zitierung/Recommended citation:}

Schimank, Uwe (2019): Coping: Entscheiden, wenn das kaum noch möglich ist. In: Leviathan, 47 (2), 192-214.

Verfügbar unter/Available at:

(wenn vorhanden, bitte den DOI angeben/please provide the DOI if available)

doi.org/10.5771/0340-0425-2019-2-192

Zusätzliche Informationen/Additional information:

Der Autor kann kontaktiert werden unter:

uwe.schimank@uni-bremen.de 
Uwe Schimank

\section{Coping: Entscheiden, wenn das kaum noch möglich ist}

Wie treffen Akteure sehr schwierige Entscheidungen, die zum einen wichtig, manchmal lebens- bzw. überlebenswichtig sind, denen sie aber zum anderen in großer Ratlosigkeit gegenüberstehen? Es geht um sehr viel: um den individuellen Lebensweg oder die Funktionstüchtigkeit einer Organisation oder die staatliche Gesellschaftsgestaltung. Doch auch wenn die Wichtigkeit der jeweiligen Entscheidungen zu einem möglichst planvollen Abwägen von Alternativen auffordert: Die Komplexität des Entscheidens ist viel zu groß, um dies realisieren zu können. Das Dilemma von Wollen, aber Nicht-Können tritt hier besonders zugespitzt auf. ${ }^{1}$

Was tun Akteure in solchen Situationen? Die Entscheidungsforschung und Diskussionen in der soziologischen Handlungstheorie geben einige Hinweise, die sich allerdings noch zu keinem schlüssigen Bild verbinden. In der Entscheidungsforschung kann man die Thematik unter den Oberbegriff der „schlecht definierten Situation“ bringen. ${ }^{2}$ Die Entscheidungsforschung hat sich hierzu zunächst auf Praktiken des Inkrementalismus als eines bewussten Planungsverzichts konzentriert, ${ }^{3}$ ist dann aber auch auf „wicked problems““4 („,bösartige“ Probleme) als weitere Zuspitzung gestoßen. Diese Probleme sind nicht lösbar, nur aushaltbar, weil eine Vielzahl Beteiligter und Betroffener mit weit auseinandergehenden Wertorientierungen und Interessen aufeinandertreffen und die Probleme zudem als Wirkungszusammenhänge nur höchst unvollständig verstanden werden. Der Klimawandel oder der Nahostkonflikt sind augenfällige Beispiele hierfür. Steht aber schon der Inkrementalismus als „science of muddling through“5 (Kunst des Sich-durchwurstelns) unter Dauerverdacht durch eine präskriptive Entscheidungstheorie, die den Planungsimperativ der Kultur der Moderne hochhält und alles, was dahinter zurückbleibt, als „Trägheit“6 missbilligt, so gilt dies erst recht für

\footnotetext{
1 Schimank 2009a, S. 56-60.

2 Kirsch 1977, S. 141-153.

3 Schimank 2005, S. 227-306.

${ }^{4}$ Rittel, Webber 1973; Danken et al. 2016 - Zitate: S. 18.

5 Lindblom 1959.

6 Dror 1964.
} 
Entscheidungspraktiken, die das Rationalitätsniveau des Inkrementalismus noch unterschreiten: Sub-Inkrementalismus kann ja nur Planungsfaulheit sein!

In der soziologischen Handlungstheorie, die bei aller Vielfältigkeit doch eine mehrheitliche Frontstellung gegenüber Rational Choice eint, spielen solche Abwertungen von Praktiken, die weit weg von rationaler Nutzenverfolgung einzustufen $(\leftarrow$ p. 192) sind, keine Rolle. Im Gegenteil haben interaktionistische, pragmatistische und praxistheoretische Handlungstheorien geradezu ein diebisches Vergnügen daran, auf Praktiken hinzuweisen, die dem wohldefinierten Modell von Rational Choice zuwiderlaufen. ${ }^{7} \mathrm{Be}-$ sonders prägnant sticht hier die Dialektik von Routine und Kreativität als Grundmotiv des pragmatistischen Handlungsverständnisses hervor: routineförmiges Agieren als Standardmodus, der aber durch Kreativität teils begleitet, teils unterbrochen wird, um unvollständige Routinen situativ einzupassen und erschütterte Routinen entweder zu reparieren oder in neue Routinen zu überführen. ${ }^{8}$ Während etwa Hartmut Esser ${ }^{9}$ als „aufgeklärter“ Vertreter von Rational Choice ein Wechselspiel von „habits“ (Gewohnheiten) und kalkulierender Nutzenverfolgung sieht, also in beiden Fällen von nur graduell unterschiedlichen "gut definierten“ Situationen ausgeht, stellen die anderen Handlungstheorien Routinen und nicht „rational choice“ für „gut definierte“ sowie Kreativität für „schlecht definierte“ Situationen heraus. Kreativität wird dabei allerdings, über die Abgrenzung von rationaler Nutzenverfolgung hinaus, kaum näher spezifiziert. Insbesondere für die pragmatistische Handlungstheorie hätte es nahegelegen, sich die reichhaltigen Befunde der empirischen Entscheidungsforschung anzuschauen, um eine fundierte Typologie der Praktiken kreativer Situationsbewältigung zu erarbeiten. ${ }^{10}$ Doch erst neuerdings gibt es - wiederum nicht auf die Entscheidungsforschung Bezug nehmende - Versuche, bestimmte Arten von Kreativität wie etwa Improvisation genauer zu fassen. ${ }^{11}$ Insgesamt achtet die soziologische Handlungstheorie wenig darauf, was Entscheiden als besonderen Typus von Handeln auszeichnet. Im Extremfall begreift sie Entscheiden nur als nachträgliche Rationalisierung von tatsächlich nicht entscheidungsförmigem Handeln - durch andere Akteure, um dem Entscheider eine

\footnotetext{
7 Breite Überblicke über soziologische Handlungstheorien geben Joas/Knöbl (2004) sowie Bonß et al. (2013); als neuere, für den Diskussionsduktus typische Auseinandersetzung mit der Rational Choice-Perspektive - hier aus praxistheoretischer Sicht - siehe, am Beispiel des Kinderkriegens, Heimerl/Hofmann (2016).

8 Joas 1992; Schubert 2009.

9 1990; 2001, S. 259-334.

10 Schimank 2009b, S. 88-90.

${ }^{11}$ Kurt, Näumann 2008; Figueroa-Dreher 2016.
} 
besondere Verantwortung für die Handlungsfolgen zuzurechnen, und im Anschluss daran oder antizipativ durch den Entscheider selbst, um sich dieser Verantwortung zu stellen. ${ }^{12}$

Vor dem Hintergrund dieser Diskussionslage geht es im Folgenden darum, das hier "Coping "13 genannte sub-inkrementalistische Entscheiden genauer daraufhin zu untersuchen,

- unter welchen Bedingungen es zum Einsatz kommt,

- welche Komponenten es aufweist und

- was daran noch rational genannt werden kann. $(\leftarrow$ p. 193)

Als empirische Anker der Argumentation dienen drei Fallbeispiele, die im ersten Teil des Beitrags zunächst je für sich dargestellt und interpretiert werden: eine Stresssituation in einer kommunalen Verwaltungsbehörde, der Überlebenskampf der griechischen Regierung angesichts der im Jahr 2009 offenbar werdenden Staatsschuldenkrise sowie die individuelle Lebensführung unter Bedingungen hochgradiger Ungewissheit. ${ }^{14} \mathrm{Im}$ zweiten Teil wird herausgearbeitet, was diese sehr unterschiedlichen Fälle mit Blick auf die Komplexität des Entscheidens und den Umgang damit gemeinsam haben; und es wird deutlich werden, dass ein so erarbeitetes generelles theoretisches Konzept von Coping als Bewältigung äußerst komplexer Situationen keine Restkategorie von sehr seltenen Extremsituationen erfasst, sondern breitere Anwendung findet.

\section{Drei Fallbeispiele}

\footnotetext{
12 Z.B. Schmitt 2018.

${ }^{13}$ Nicht in Anknüpfung an den psychologischen Sprachgebrauch, der vor allem ein Coping mit „kritischen Lebensereignissen“ thematisiert (Filipp, Aymanns 2010), und auch nicht an die psychologische Entscheidungsforschung von Irving Janis und Leon Mann (1976; 1977) anschließend, die als Coping alle Arten von Reaktionsmustern auf ein Entscheidungsproblem benennen. Hier wird eher eine Sprachregelung aufgegriffen, die im Englischen in Buchtiteln wie „Coping with the Euro crisis" zum Ausdruck kommt: Coping als Bewältigung eines besonders schwierigen Problems, die mit diesem gerade so eben, oft nur "mit Ach und Krach", zurechtkommt - wobei bedauernd mitschwingt, dass die Bedeutung des Problems eigentlich eine planvollere Umgangsweise erfordert hätte.

${ }^{14}$ Dass keiner der Fälle wirtschaftliches Entscheiden behandelt, ist dem Zufall der verfügbaren Daten geschuldet und sollte nicht so gelesen werden, dass in der Wirtschaft mehr Rationalität herrscht. Die meisten Befunde der Forschung zur begrenzten Rationalität organisationalen Entscheidens sind an Unternehmen gewonnen worden (siehe Hodgkinson und Starbuck 2008); auf individueller Ebene ist das Pendant die verhaltensökonomische Forschung zum Konsum- und Geldanlageentscheiden (Beck 2014).
} 
Zur Einordnung der drei Beispiele sei zunächst ganz knapp der allgemeine entscheidungstheoretische Bezugsrahmen umrissen, in dem Coping hier verortet wird. ${ }^{15}$ Entscheiden ist in diesem Verständnis ein Alternativen bedenkender Modus des Handelns, Abwägen ist der Kern von Entscheiden - im Unterschied vor allem zu Routinen, bei denen bei Vorliegen eines auf sie passenden vordefinierten Anlasses die Akteure sogleich und gleichsam „bedenkenlos“ wissen, was zu tun ist. ${ }^{16}$ Das entscheidungsförmige Bedenken von Alternativen geschieht entsprechend einer prozeduralen Rationalität, deren Befolgung Ergebnisrationalität wahrscheinlicher macht: Möglichst „gutes" Entscheiden kann eine möglichst "gute" Entscheidung zwar niemals garantieren, aber doch in Aussicht stellen.

Perfekt rationales Entscheiden hieße prozedural:

- in sachlicher Hinsicht eine vollständige Erfassung und Verarbeitung der relevanten Informationen, $(\leftarrow$ p. 194)

- in sozialer Hinsicht eine Überführung der unterschiedlichen Perspektiven und daraus hervorgehenden Problemdeutungen aller Entscheidungsbeteiligten und -betroffenen in eine allgemein anerkannte Sicht der Dinge,

- und in zeitlicher Hinsicht hinreichend Zeit für eine vollständige Informationsverarbeitung und die Erarbeitung einer allgemein anerkannten Sicht der Dinge.

Dem steht die Komplexität von Entscheidungssituationen entgegen:

- in sachlicher Hinsicht als Fehlen wichtiger Informationen oder als nicht verarbeitbarer Informations-Overload,

- in sozialer Hinsicht als Erwartungsunsicherheit bezüglich des Handelns von Gegenübern und als Konflikte mit einflussstarken Gegenübern,

- sowie in zeitlicher Hinsicht als Zeitknappheit.

Der Entscheidungsmodus der Planung kommt perfekter Rationalität am nächsten. Er bemüht sich um eine zeitlich, sachlich und sozial ambitionierte Art des Entscheidens: langfristig angelegt, umfassend und kreativ in der Informationsverarbeitung und Alternativensuche und dabei auf einen möglichst breiten Konsens ausgerichtet. Die Voraussetzung dafür, dass dieser Modus erfolgversprechend zum Zug kommen kann, ist allerdings eine vergleichsweise geringe Komplexität der Entscheidungssituation. Doch

\footnotetext{
15 Schimank 2005.

16 Luhmann 1964.
} 
diese Voraussetzung ist oftmals umso weniger gegeben, je wichtiger das Entscheidungsproblem ist - woraus sich das Entscheidungsparadox ergibt: Je wichtiger eine Entscheidung ist, desto höher ist tendenziell ihre Komplexität, und desto weniger rational kann das Entscheiden sein. Planung wird also zumeist gerade dann unmöglich, wenn sie dringend erforderlich wäre.

Und was dann? Ich präsentiere im Folgenden drei Fallstudien von Entscheidungshandeln, das unübersehbar alles andere als planvoll ist - in vielen Zügen geradezu das genaue Gegenteil. Alle drei Fälle stellen hochgradig komplexe Entscheidungssituationen dar; und es tritt jeweils einer der drei zentralen Akteurstypen der Moderne auf: Individuen, Organisationen und Staaten. ${ }^{17}$ Ich beschreibe zunächst jeden der Fälle für sich: Worum geht es beim jeweiligen Entscheiden? Was ist das Komplexitätsprofil der Entscheidungssituationen? Und was tun die Entscheider? Anschließend arbeite ich in einer vergleichenden Zusammenschau Coping als analytisches Konzept für solches Entscheiden heraus und zeige dabei auf, in welchen Hinsichten noch von einer RestRationalität dieses Entscheidens gesprochen werden kann.

\section{Eine Behördenleiterin im Organisationsstress ${ }^{18}$}

In einer deutschen kommunalen Behörde mit Publikumsverkehr kommt an einem Tag Organisationsstress auf, weil zu länger- und mittelfristigen ungünstigen Rahmenbedingungen noch akute Tagesprobleme hinzutreten: $(\leftarrow$ p. 195)

- eine Organisationsaufgabe, die häufige Konflikte mit den Klienten - hier „Kunden" genannt - in sich birgt und eine äußerst diffizile, gerade wieder geänderte Rechtslage;

- eine schwierige Umorganisationsphase;

- personelle Engpässe, zusätzlich - auch infolgedessen - ein hoher Krankenstand auf der Arbeitsebene;

- eine unbesetzte Leitungsposition, und nur zwei von sechs Teamleiterpositionen besetzt;

\footnotetext{
17 Meyer, Jepperson 2000.

18 Für dieses Beispiel greife ich auf eine Hausarbeit im Master-Studiengang „Entscheidungsmanagement" an der Universität Bremen zurück; die Autorin möchte anonym bleiben.
} 
- am betreffenden Tag ein auswärtiger Termin des stellvertretenden Leiters und eine Erkrankung eines Teamleiters.

Die einzige verbleibende Teamleiterin musste - als dafür nicht eingearbeitete - kommissarische Leiterin der Behörde dafür sorgen, „ dass der Dienstbetrieb weiterlief.“ Das hieß, „die Sachbearbeiter aus den Teams bei sich ergebenden rechtlichen Fragestellungen zu unterstützen, Beschwerden entgegenzunehmen und Notfälle irgendwie noch unterzubringen."

Ein aufgebracht auf sie einredender Notfallkunde saß gerade bei ihr im Zimmer. Während sie überlegte, zu welchem Sachbearbeiter sie ihn schicken könnte, klingelte das Telefon: Ein Mitarbeiter des Servicepoints hatte eine Notfallkundin, die unbedingt bedient werden wollte. Auf dem Weg zum Servicepoint - ihr eigener Kunde wurde auf den Flur zum Warten geschickt - wurden ihr von Mitarbeitern zwei weitere Kundenbeschwerden mitgeteilt. Am Servicepoint konnte die Leiterin der Kundin nur dasselbe wie die Sachbearbeiterin mitteilen: Sie müsse einen Termin vereinbaren oder als Laufkundin lange warten. Die anderen beiden Beschwerden wurden an die Mitarbeiter zurückgegeben, die die Kunden beschwichtigen sollten.

Auf dem Rückweg ins Büro sprachen drei Sachbearbeiter die Leiterin mit Kundenfragen an; sie musste um Geduld bitten. Ihr eigener Kunde war mittlerweile gegangen ein Problem weniger! Auf ihrem Tisch lag ein Zettel mit Verweis auf einen weiteren dringenden Notfallkunden. Sie schaute auf ihrem Computer ins Terminvergabesystem und fand tatsächlich einen freien Termin, der sich wegen Nichterscheinens eines Kunden plötzlich aufgetan hatte; doch vor Ort beim betreffenden Mitarbeiter erwies sich, dass schon ein anderer Kunde vorgezogen worden war. Eine im selben Zimmer sitzende andere Sachbearbeiterin erklärte sich aber spontan bereit, diesen Notfallkunden zu bedienen, weil sie ihren Terminkunden schneller hatte abfertigen können. Wieder ein Problem weniger: aber immer noch die drei anderen anhängigen Kunden, wenn nicht mittlerweile weitere zu entscheidende Fragen dazugekommen waren. Und so ging es den ganzen Tag weiter.

Diese Entscheiderin musste mit all ihren Entscheidungen „irgendwie über den Tag kommen“ und hoffen, dass es „am kommenden Tag schon anders“ aussehen könnte. Eine chronische Personalknappheit verknüpfte sich mit weiterem akuten Personalausfall, was der ohnehin gegebenen sachlichen und sozialen Komplexität des Entscheidens - der schwierigen Rechtslage und den konfliktträchtigen Kundenbeziehungen - 
auch noch Zeitdruck hinzufügte, weil zu wenige Mitarbeiter zu viele Entscheidungen zu treffen hatten. Die kommissarische Leiterin konnte lediglich versuchen, das Tagesgeschäft so zu managen, dass die vielen Störungen nicht in einem großen Knall endeten, und dort, wo der größte Druck herrschte und sich $(\leftarrow$ p. 196) situative Gelegenheiten zur Abfertigung von Einzelfällen ergaben, diese Chancen kurzerhand zu ergreifen. Eine Prioritätensetzung der Fälle entsprechend deren Bedürftigkeit konnte nicht stattfinden; wer gerade im passenden Moment in der Nähe war und am lautesten auf sich aufmerksam machte, wurde bedient. Es handelte sich um Krisenmanagement der zugespitzten Form. An Planung war nicht zu denken. Die Gegebenheiten erlaubten nicht einmal kurzes Nachdenken über einen Notfallplan für den akuten Personalengpass. Die Leiterin konnte nur noch eine gewisse Entscheidungsfähigkeit der Organisation aufrechterhalten, wenn auch um den Preis einer nicht unerheblichen Fehlerquote. $^{19}$

\section{Der griechische Finanzminister in der Staatsschuldenkrise ${ }^{20}$}

Im Oktober 2009 löste in Griechenland die sozialistische PASOK die bis dahin regierende konservative Partei Nea Dimokratia ab, und Giorgos Papakonstaninou (im Weiteren: GP) wurde Finanzminister unter Regierungschef Giorgos Andreas Papandreou (im Weiteren: GAP). Schon am zweiten Tag im Amt erfuhr GP, was niemand in der neuen Regierung in diesem Ausmaß für möglich gehalten hätte: „Griechenland war in großer Gefahr. Das öffentliche Defizit war wie ein ungebremster Zug, mindestens zwei Mal höher, als die Vorgängerregierung eine knappe Woche zuvor offiziell angegeben hatte." ${ }^{21}$ Spätestens zu diesem Zeitpunkt trat die Krise der griechischen Staatsfinanzen, die sich zu einer Krise der Europäischen Union (EU) ausweiten sollte, in ihre akute Phase. Als GP knapp zwei Jahre später im Juni 2011 von GAP unter hohem öffentlichen Druck entlassen wurde, war die Krise keineswegs beendet. Sie zog sich vielmehr noch Jahre hin. Erst vor nicht allzu langer Zeit konnte ein langer

\footnotetext{
19 Sie würde vermutlich selbst, wenn sie das Geschehen nochmals in Ruhe Revue passieren ließe, Manches beim nächsten Mal anders machen.

20 Die Fallschilderung beruht auf dem Selbstbericht von Giorgos Papakonstantinou (2017)

- Seitenzahlen in diesem Abschnitt beziehen sich auf dieses Buch. Da der Fall hier nur exemplarisch und heuristisch als Anregung für allgemeinere theoretische Überlegungen herangezogen wird, kommt es nicht darauf an, ob alles Geschilderte wahrheitsgemäß ist und welche anderen Perspektiven auf das Geschehen es gibt.
}

${ }^{21}$ Papakonstantinou 2017, S. 31. 
Konsolidierungsprozess zumindest insoweit Erfolge zeitigen, dass etwa die Ratingagentur Fitch das Rating der Kreditwürdigkeit des griechischen Staates, das dessen Chancen entscheidend bestimmt, weitere Kredite auf dem Finanzmarkt zu erhalten, von zuletzt „B-, auf „B“ angehoben hat. ${ }^{22}$ Das ist zwar keine „A“-Note, jedoch deutlich besser als "CCC“23 auf dem Tiefpunkt der Krise.

Die Entscheidungssituation, in die GP durch das beim Amtsantritt übernommene und von den Vorgängern verheimlichte Finanzproblem versetzt wurde, war in allen drei Dimensionen - einschließlich deren Wechselwirkungen - durch eine extrem hohe Komplexität gekennzeichnet. In sachlicher Hinsicht war die griechische Staatsschuldenkrise auf viele Faktoren zurückzuführen, die in ganz verschiedenen gesellschaftlichen Sphären - von der Wirtschaft über das Gesundheits- und Bildungssystem $(\leftarrow \mathbf{p}$. 197) bis zum Militär und Familienleben - angesiedelt waren und mannigfaltige, sich negativ aufschaukelnde Wirkungsverkettungen hervorbrachten: manche davon bekannt, andere opak. Bei einigen Wirkungsverkettungen hatte man Zugriff auf Stellschrauben, bei anderen nicht. Man hatte es insgesamt mit einer zum einen höchst unvollständig verstandenen Problematik zu tun, in die man zum anderen selbst da, wo man sie halbwegs verstand, oft kaum eingreifen konnte.

Das verband sich in sozialer Hinsicht mit einer Akteurkonstellation, innerhalb derer GP agierte, die durch extrem divergierende, oft diametral entgegengesetzte Interessen und Forderungen der involvierten nationalen und internationalen politischen und anderen Akteure charakterisiert war. Viele von innen konnten in bestimmten Hinsichten als Vetospieler auftreten und taten dies auch. Bereits innerhalb des eigenen Ministeriums traf GP auf einflussreiche Gegenspieler. Das setzte sich innerhalb der Regierung und Regierungspartei fort. National gab es eine starke parlamentarische und außerparlamentarische Opposition zahlreicher Gruppen mit Spezialinteressen, die gegen viele Maßnahmen des sowieso traditionell unbeliebten Finanzministers größere Teile der Bevölkerung aufzuwiegeln in der Lage waren. Eine kleine Reminiszenz der Gespräche, die GP immer wieder mit seinen Ministerkollegen zu führen hatte: „Einige der verlassenen Militärstandorte im Land schließen? Sehr schwer. Die Bezahlung von Überstunden in öffentlichen Krankenhäusern einschränken, oder die garantierten Profitmargen von Apothekern reduzieren? Unmöglich. Staatlichen Unternehmen mitteilen,

\footnotetext{
22 Westdeutsche Zeitung, 17.2.2018: www.wz.de/.../ratingagentur-fitch-sieht-griechenlandauf-erholungskurs-1.2623213

${ }^{23}$ Papakonstantinou 2017, S. 265.
} 
dass sie sich umzustellen und die Kosten zu reduzieren hatten, ansonsten ...? Nicht wirklich. Die Anzahl der Einstellungen von Absolventen der Polizei- und Militärakademie verringern? Ist das dein Ernst?"24

Noch stärker waren aber die Einflüsse von Akteuren, die außerhalb Griechenlands positioniert waren: von der EU und der Europäischen Zentralbank (EZB) bis zu Internationalem Währungsfond (IWF), Weltbank, Rating-Agenturen und internationalen Banken und Investmentfonds. Der griechische Finanzminister war nur ein sehr kleiner Player in dieser globalen Konstellation, der wenig Unterstützer oder Verbündete, aber viele oftmals weit einflussreichere Widersacher hatte und es unmöglich allen oder auch nur den wichtigsten Gegenübern halbwegs recht machen konnte. In zeitlicher Hinsicht schließlich befand sich GP in einer Situation durchgängig hoher Zeitknappheit, die immer wieder extreme Deadlines wie diese setzte: „[U]m einen Bankrott zu vermeiden, hatten wir etwas mehr als eine Woche Zeit, eine Vereinbarung auszuhandeln."25 und zwar mit einer Mehrzahl von Gegenübern, die jeweils ganz eigene Vorstellungen hatten. Zudem galt in verschiedenen Phasen: „Das Ganze drohte zu einer sich selbst erfüllenden Prophezeiung zu werden."26 Eine solche eigendynamische Problemeskalation über die Zeit hätte den griechischen Staatsbankrott hervorrufen können, wenn herabgestufte Ratings den Zugang zu Finanzmitteln erschwert hätten, was die finanziellen Schwierigkeiten des griechischen Staats weiter verschärft hätte, was zu einer weiteren Herabstufung ( $\leftarrow$ p. 198) geführt hätte, usw.: „Wenn die Märkte daran glauben, dass die Schulden tragfähig sind, dann leihen sie weiterhin Geld, so dass die Schulden de facto tragfähig sind - ein Zirkelschluss. Wenn sie sich hingegen entscheiden, dass die Schulden nicht tragfähig sind, leihen sie dir kein Geld mehr und die Schulden werden untragbar." 27

Angesichts all dessen hat sich GP zunächst einmal zur Einsicht in die Realitäten entschieden. Die nationale Statistikbehörde wurde von ihm angewiesen, die geschönten Zahlen zur Staatsverschuldung zu revidieren und möglichst schnell das wahre Ausmaß zu beziffern. Diese Zahlen wurden nicht nur den europäischen Partnerregierungen, der EU und dem EZB mitgeteilt, sondern auch der eigenen Bevölkerung, um den ganzen Ernst der Lage zu vermitteln. Einsicht bedeutete im nächsten Schritt, sich dafür

\footnotetext{
24 Ebd., S. 56

25 Ebd., S. 141.

26 Ebd., S. 84.

27 Ebd., S. 237.
} 
zu entscheiden, die in den Folgemonaten von der EU und dem IWF auferlegten Sparmaßnahmen und weiteren Reformen zu akzeptieren, weil nur so die Bereitschaft dieser Akteure, mit Krediten auszuhelfen, gesichert werden konnte. In einer Serie von Maßnahmepaketen setzte man „die drastischsten Ausgabenkürzungen und Steuererhöhungen, die je von einer griechischen Regierung angekündigt wurden", durch. ${ }^{28}$ Hinzu kamen Privatisierungen, Arbeitsmarktreformen und der Abbau von Privilegien verschiedenster gesellschaftlicher Gruppen - wie nicht anders zu erwarten unter heftigstem Protest. Diese zwei Nicht-Entscheidungen - Nicht-weiter-wie-bisher und Sichnicht-widersetzen - steckten den Rahmen dessen ab, was GP darüber hinaus tun konnte. Sich nicht länger zu widersetzen schloss ein, dass man selbst viele Maßnahmen entschied, die generelle Zielvorgaben der Geldgeber umsetzten. Diese Entscheidungen hatten zumeist durchaus die zeitliche Reichweite größer angelegter Planungen - etwa Anfang 2010 als „Drei-Jahres-Plan, (...) um das Defizit unter die Schwelle von drei Prozent des BIPs zu bringen.“29 GP erläutert: „Das war eine politische Blaupause für die kommenden drei Jahre, mit Zeitplänen, mit spezifischen und durchkalkulierten Maßnahmen sowie mit einem Überwachungsmechanismus, um sicherzustellen, dass die eingegangenen Verpflichtungen auch zu konkreten Handlungen führten. Sollte dies nicht der Fall sein, würde die ultimative Sanktion folgen: die Verweigerung finanzieller Unterstützung und eine damit verbundene staatliche Insolvenz."30 Was hier als Planung konzipiert und auch zu vollziehen versucht wird, ist freilich, wie der Schlusssatz zeigt, nur in auferlegter Form machbar. Der Plan muss GP, auch wenn er mit inm übereinstimmt, von Gegenübern diktiert werden, die aufgrund ihrer Übermächtigkeit die Komplexität in der Sozialdimension überwinden können. Auch diese Akteure stehen freilich in der Sach- und der Zeitdimension vor der extrem hohen Komplexität und können nur hoffen, dass die Pläne halbwegs in die richtige Richtung gehen.

Weil diesbezüglich erhebliche Ungewissheiten bestanden, musste die Planung mit inkrementalistischen Flexibilitäten flankiert werden, die GP die Möglichkeit zum $(\leftarrow \mathbf{p}$. 199) eigenen Mitentscheiden boten. Er konnte den Gegenübern von EU, EZB und IWF in sachlicher und sozialer Hinsicht gewisse Zugeständnisse auf dem Weg - anstatt einer strikten Planerfüllung - abringen, indem er unter Verweis auf das bereits Geleistete und die sich zeigenden Effekte an deren Einsicht in das Machbare und Zumutbare

\footnotetext{
28 Ebd., S. 110.

29 Ebd., S. 48.

30 Ebd., S. 161.
} 
appellierte. Sparauflagen wurden so ein Stück weit nach unten korrigiert, und Härten für bestimmte Gruppen wie etwa die Rentner wurden gemildert, um die Proteste nicht eskalieren zu lassen und der Regierung die parlamentarische Mehrheit zu sichern. Wo die Gegenüber nicht über die Planvorgaben mit sich reden ließen, konnten zumindest zeitliche Streckungen der Zielerreichung nachverhandelt werden.

Was hat GP erreicht? Man könnte zunächst meinen, dass er sehr erfolglos agierte, denn als er von GAP entlassen wurde, befand sich das Rating der Kreditwürdigkeit des griechischen Staates auf dem schon angeführten Tiefstand. In einer Zwischenbilanz der ersten Phase seiner Amtszeit hält GP Mitte 2010 durchaus zufrieden fest: „Wir hatten es geschafft. Ein Desaster war vermieden worden, Griechenland würde nicht das erste Land in der Eurozone werden, das nicht mehr in der Lage wäre, seine Schulden zurückzuzahlen." Was damit „geschafft" war, sagt er gleich im nächsten Satz: „Wir konnten den nächsten Tag überleben und weitere Schlachten schlagen - aber die wahre Odyssee hatte gerade erst begonnen." "31 Die Problematik war also, wie inm bewusst war, alles andere als abgehakt. Doch immerhin hatte man bis jetzt ein ultimatives Scheitern abgewendet.

Die Behördenleiterin hatte die durchaus begründete Hoffnung, dass es bereits am nächsten Tag deshalb besser aussehen dürfte, weil dann weitere Leitungspersonen wieder vor Ort im Dienst sein würden. Auch GP konnte kurz nach der zitierten Einschätzung Zeichen der Besserung registrieren: „[]]n jenem Sommer hatten wir alle den Eindruck, dass Bewegung in die Sache gekommen war. Die Zahnräder drehten sich wieder langsam, die Maschine begann zu arbeiten, Vernunft kehrte in ein wildgewordenes System zurück. "32 Doch bald zeigte sich, dass es nicht kontinuierlich weiter bergauf ging. Als die definitiv revidierten Schuldenstände des griechischen Staates für die Vorjahre bekannt wurden, wurde klar, dass die Sparbemühungen noch drastischer ausfallen müssten, um die vereinbarten Ziele zu erreichen - „was noch nie zuvor irgendwo auf der Welt gelungen ist." ${ }^{33}$ Doch wurde Griechenland auch Mut gemacht: „[W]ir könnten wenigstens mit der Verlängerung der offiziellen Kreditlaufzeiten rechnen - das würde uns helfen, an die Kapitalmärkte zurückzukehren. Und wir dürften auf eine

\footnotetext{
31 Ebd., S. 181.

32 Ebd., S. 184.

33 Ebd., S. 212.
} 
wirtschaftliche Wende im Frühjahr oder Sommer des kommenden Jahres rechnen.“34 Das Entscheidungshandeln durchlief also ein Auf-und-ab.

Dass GP seinem Nachfolger schließlich eine schlechter bewertete staatliche Kreditwürdigkeit übergab, als er übernommen hatte, heißt somit nicht unbedingt, dass sich die Problematik der Staatsfinanzen insgesamt verschlechtert hatte. Denn inzwischen war die Problematik ganz oben auf der Agenda und wurde in einer kollektiven $(\leftarrow \mathbf{p}$. 200) Anstrengung vieler Akteure bearbeitet, anstatt sich untergründig weiter zu verschärfen; und dass Erfolge angesichts der extrem komplexen Lage Zeit brauchen würden, ist ebenfalls evident. Auch wenn es schwierig sein dürfte, im Rückblick zu beurteilen, wie groß der Anteil von GP an der mittlerweile eingetretenen Verbesserung war, muss man inm als Minimum zurechnen, dass er in seiner Amtszeit den Staatsbankrott vermieden und die längerfristige Bearbeitung der zugrundeliegenden Problematik auf den Weg gebracht hat.

\section{Lebensführung unter Ungewissheit}

Während es im ersten Fall um eine akute Notsituation und im zweiten um eine chronifizierte Notlage ging, werden nun im dritten Fall Entscheidungssituationen angesprochen, bei denen die Akteure davon ausgehen müssen, dass es sich um einen Dauerzustand handeln könnte - der allerdings nicht ständig hochproblematisch sein muss. Es geht um Entscheidungen, die im Lebensverlauf von Individuen anfallen: Berufsoder Studienwahl, Entscheidungen für oder gegen bestimmte Arbeitsangebote, für oder gegen Lebenspartner, Wohnorte, Hausbau, Kinderkriegen, Geldanlagen auf dem Finanzmarkt u.ä. Bei derartigen Entscheidungen gibt es in der westlich geprägten „Weltkultur“35 die hegemoniale Vorgabe, planvoll zu agieren: langfristig vorausschauend sowie nach links und rechts schauend ein möglichst breites Spektrum von Alternativen zu sondieren und nach umfassender Bewertung die beste auszuwählen, anstatt einfach „in den Tag hinein“ zu leben. Ich wende mich nun - anders als bei den ersten beiden Fällen - keinen konkreten Einzelfällen zu, sondern Falltypen in Gestalt von drei Lebenslagen, in denen diesem Planungsimperativ nicht gefolgt werden kann, weil jeweils zu große Ungewissheiten herrschen.

\footnotetext{
35 Meyer 2005.
} 
Eine Extremgruppe sind marginalisierte Arme, etwa in Brasilien. ${ }^{36}$ Sie arbeiten als Straßenverkäuferinnen oder Hausangestellte, in manchmal von Tag zu Tag wechselnden Tagelöhnertätigkeiten, oder sie beschaffen sich durch Prostitution, Kleinkriminalität oder Betteln das Geld zum täglichen Überleben. Sie leben in instabilen Familienverhältnissen in den Armenvierteln. Häufig wissen sie heute nicht, was morgen auf sie zukommt und was sie dann tun werden. Diese „kontinuierliche Diskontinuität“ bewirkt, dass "die Zukunft weit von der Gegenwart entfernt und beinahe abgetrennt“ ist. ${ }^{37}$ Lebensführung ist für diese Armen - wie abgemildert auch für Obdachlose in westlichen Gesellschaften - „gefangen im Moment“" ${ }^{38}$, eine Planung über den Tag hinaus entbehrt jeder Grundlage und findet daher nicht statt. Allerhöchstens vage Zukunftsträume, manchmal religiös bestärkt, reichen über Fatalismus hinaus, bleiben aber Tagträume, denen keinerlei Gestaltungskraft im „wirklichen Leben“ innewohnt. Routinen dominieren die alltägliche Lebensführung, und wenn - was oft genug geschieht - irgendwelche Störungen auftreten, kann $(\leftarrow$ p. 201) nur aus dem Moment heraus irgendetwas, was gerade möglich ist, getan werden: „Beispielsweise müssen Straßenverkäuferlnnen den Standort oder die Artikel wechseln, wenn sie nicht genug verdienen."39 Oder sie müssen sich einen weiteren Job suchen und noch länger arbeiten. Eine über den Tag hinaus reichende Lebensführung, die solche Ungewissheiten reduziert und wenn schon nicht Planbarkeit, so doch zumindest Kalkulierbarkeit schafft, ist unerreichbar.

Friedhelm Guttandin ${ }^{40}$ hat in einer agrarisch geprägten Provinzstadt in Paraguay weniger extreme, aber dennoch deutlich ausgeprägte Ungewissheiten und darauf ausgerichtete Praktiken der Lebensführung größerer Bevölkerungsgruppen, auch vieler Mittelschichtsangehöriger, entdeckt. Die wichtigsten Quellen der dortigen Ungewissheiten sind:

- unberechenbare Naturgewalten und -zyklen wie Überschwemmungen oder Missernten;

- die Schwankungen der Weltmarktpreise für die produzierten und exportierten Agrargüter;

\footnotetext{
${ }^{36}$ Zum Folgenden siehe Florian Stoll 2012, S. 311-349.

37 Stoll 2012, S. 332-333.

38 Stoll 2012, S. 331.

39 Stoll 2012, S. 331.

401996.
} 
- „die Willkür der Mächtigen in Staat und Politik“41 und die „Unberechenbarkeit der Rechtsordnung"42;

- die Fragilität der guten Beziehungen, die man zu vielen anderen - nicht nur den „Mächtigen“ - pflegen muss.

Ob das traditionell starke Vertrauen in den Rückhalt durch die eigene Familie und engere Verwandtschaft diese Ungewissheiten ausgleichen kann, wird immer fraglicher nicht zuletzt, weil vom Arbeitsmarkt auferlegte räumliche Mobilität zunehmend Verwandtschaften auseinanderreißt, so dass keine Unterstützung in schwierigen Situationen mehr gewährt werden kann.

Guttandin beobachtet ein Wechselspiel von zwei Arten des Umgangs mit solchen Ungewissheiten. Zum einen muss man sich in „langen Phasen des Wartens“43 in Geduld üben, bis sich die Ungewissheiten klären und man weiß, was man tun könnte. Zum anderen kommen aber, manchmal urplötzlich, Momente, in denen man ad hoc eingreifen kann und „schnell und entschlossen“ 44 zupacken muss. Wenn man das durch zu langes Überlegen verpasst, kann die Gelegenheit schon wieder vorbei sein, und das Warten beginnt erneut. „Irgendwie-Zurechtkommen, Ins-Laufen-Bringen, Flickschusterei, (...) ,Gut genug für den Augenblick'“: Das sind Devisen dieses Wechselspiels von langem Warten und schnellem Zuschlagen. ${ }^{45}$

Hinsichtlich der zugrundeliegenden Lebenshaltung dieser paraguayischen Provinzstadtbewohner zieht Guttandin ${ }^{46}$ einen Vergleich zu Helmut Schelskys Porträt $(\leftarrow \mathbf{p}$. 202) der „skeptischen Generation“ der westdeutschen Nachkriegsgesellschaft, die nach Nationalsozialismus und Zweitem Weltkrieg „zutiefst von der planerischen Ohnmacht des Menschen gegenüber großen politischen und sozialen Kräftekonstellationen überzeugt" gewesen sei und sich deshalb in ihren Ansprüchen, die Gesellschaft oder auch nur das je eigene Leben planvoll gestalten zu können, enorm zurückgenommen hatte: Erst einmal sichern, was man hat, und ansonsten keine „großen Sprünge“ machen, sondern sich mit Nahzielen begnügen. Nach der „skeptischen Generation“ kamen in Deutschland die „Technokraten“ und die „68er“, zwei bei allen sonstigen Unterschieden gleichermaßen große Gestaltungsambitionen hegende Gruppen -

\footnotetext{
41 Guttandin 1996, S. 66.

42 Guttandin 1996: S. 140.

43 Guttandin 1996, S. 37.

44 Ebd.

45 Guttandin 1996, S. 66.

46 1996, S. 163.
} 
hinsichtlich der Gesellschaft wie der je eigenen Lebensführung. Als diese Ambitionen sich zerschlugen, wofür in der Jugendkultur Ende der 1970er Jahre die "no future“Haltung der Punks stand, setzte sich erneut Planungsskeptizismus in einer immer komplexer gewordenen Welt durch. Cornelia Koppetsch ${ }^{47}$ porträtiert auf dieser Linie heutige junge Erwachsene in Deutschland als neue „skeptische Generation“, die gar nicht so weit von der improvisierten Lebensführung der paraguayischen Provinzstadtbewohner weg ist.

Für Koppetsch ${ }^{48}$ sind es vor allem die Dynamiken und Turbulenzen des globalisierten und politisch nicht länger gebändigten Kapitalismus, denen sogar sehr gut ausgebildete junge Arbeitnehmerinnen in dessen westlichen Zentren dergestalt ausgesetzt sind, „dass die Möglichkeiten einer selbstbestimmten Lebensführung abnehmen, statt zu wachsen." Wenn jemand z.B. von seinem Arbeitgeber für mehrere Jahre ins Ausland versetzt wird, was beruflich im Falle der Bewährung dort durchaus zu einem Karrieresprung werden kann, aber in einer Familienkonstellation mit einem ebenfalls die eigene Berufskarriere verfolgenden Ehemann und schulpflichtigen Kindern eine starke Irritation mühsam austarierter Arrangements von Beruf, Partnerschaft und Bildungslaufbahn der Kinder darstellt, dann zeigt sich sehr schnell: Eine planvolle Bewältigung dieser Irritation müsste entsprechende Informationsbeschaffung u.a. über Berufsmöglichkeiten des Mannes und schulische Angebote für die Kinder im betreffenden Land, Verhandlungen - etwa mit dem Arbeitgeber des Mannes über dessen Beurlaubung mit Rückkehrgarantie - und gemeinsame Abwägungen und Aushandlungen innerhalb der Familie beinhalten. Eine Irritationsbewältigung durch ein planvolles nachhaltiges Neuarrangement der Lebensführung überstiege aber in ihrer Aufwendigkeit - was Informationsverarbeitung, Abstimmung und Konfliktbewältigung unter Bedingungen von manchmal großem Zeitdruck und begrenzten Ressourcen anbelangt - schnell das, was machbar ist. Genau deshalb findet man heutzutage, gerade unter Jüngeren, zwar durchaus eine Sehnsucht nach mehr Planbarkeit des eigenen Lebens, aber zugleich sehr zurückgenommene Planungsbemühungen oder gar einen weitreichenden lakonischen Planungsverzicht. ${ }^{49}(\leftarrow$ p. 203)

\footnotetext{
47 2013, S. $109-120$.

48 2013, S. 116.

49 Schimank 2015.
} 
Koppetsch ${ }^{50}$ schlussfolgert: „Es wäre für die meisten jungen Erwachsenen heute fatal, einem ,Lebensplan' zu folgen oder an einer persönlichen Selbstentfaltung festzuhalten, wenn diese den äußeren Umständen oder Erwartungen anderer entgegenstehen." Stattdessen gelte: „Der Einzelne muss sein Leben so gestalten, dass er in der Lage ist, ,im Rennen zu bleiben“." Das wiederum heiße vor allem, man müsse „seine Ausgangssituation verbessern und im richtigen Moment zugreifen."

Zwischen der brasilianischen Straßenverkäuferin und dem sehr gut verdienenden ITSpezialisten in Berlin bestehen zweifellos beträchtliche Unterschiede, was das Ausmaß an „agency“ bei der Gestaltung der eigenen Lebensumstände und Lebensführung anbelangt. Doch beide haben gemeinsam, dass Lebensplanung in einem anspruchsvollen Sinne nicht möglich und daher auch nicht ratsam ist. Stattdessen herrscht bei beiden Improvisation vor - und zwar nicht in der aktiv-schöpferischen, freiwillig gesuchten Variante wie z.B. in der Jazzmusik, sondern als auferlegte reaktive Schadensbegrenzung und Nutzung kleiner Verbesserungschancen. ${ }^{51}$

\section{Vier Komponenten von Coping}

Den drei Fallbeispielen ist gemeinsam, dass Akteure es mit hochkomplexen Entscheidungssituationen zu tun haben, in denen es kaum etwas zu gewinnen gibt, sondern man nur versuchen kann, erst einmal über die Runden zu kommen und Schlimmeres zu verhüten. Dies sind nicht die Stunden, in denen Entscheider durch erfolgreich aufgehende Pläne glänzen können; sie können sich genau umgekehrt nur noch darum bemühen, als Entscheider nicht ganz von der Bildfläche zu verschwinden.

Fragt man fallübergreifend, was die Entscheider in den drei Fällen eigentlich tun, um mit der sehr hohen Entscheidungskomplexität umzugehen, erkennt man jeweils sehr deutlich drei von vier Komponenten dessen, was ich als Coping herausarbeiten will; die vierte Komponente kommt eher andeutungsweise zum Ausdruck.

Erstens fällt auf, dass die Behördenleiterin, der Finanzminister und die in biographischer Ungewissheit Lebenden ihr Handeln nicht an klaren Zielen ausrichten. Anstelle eines systematisch geordneten Katalogs präziser Ziele einschließlich einer daraus

\footnotetext{
50 2013, S. 116 ; S. 120.

51 Siehe Guttandins (1996, S. 14-16) Unterscheidung von „Improvisation auf hohem Niveau“ und „substantieller Improvisation“.
} 
ableitbaren Schrittfolge der Zielverfolgung sehen diese Entscheider das jeweilige eigene Tun im Rahmen vager, sehr Vieles offen haltender Zielkorridore wie „irgendwas tun, um die EZB bei Laune zu halten, ohne die eigenen Rentner gegen sich aufzubringen“ oder „endlich anfangen mit dem Schreiben der Dissertation - wozu auch immer der Doktortitel mal gut sein wird“. Wenn nötig, stellt man selbst diese Korridore zur Disposition und tauscht sie gegen andere aus wie z.B. „vielleicht doch jenseits der Wissenschaft einen Job suchen“. Es liegt, verglichen mit Planung und ihren langfristig und umfassend angelegten Zielkatalogen, eine weitgehende Zielausdünnung vor: $(\leftarrow$ p. 204)

- Die Behördenleiterin muss durchaus formulierte Zielgrößen - sachgerechte, effiziente und faire Behandlung der Klienten, was dann weiter z.B. als maximale Wartezeit von Laufkunden oder maximale Fehlerquote von Bescheiden operationalisiert sein könnte - allesamt ignorieren, weil all ihr Streben nur noch darauf ausgerichtet ist, den Tag ohne innere Zerwürfnisse oder schlechte Presse zu überstehen. Dieser Vermeidungsimperativ, kein positiv spezifiziertes Ziel leitet ihr Entscheiden.

- Der griechische Finanzminister orientiert sich ganz ähnlich vor allem daran, den Spagat hinzubekommen, weder die Gunst der externen Geldgeber zu verspielen noch das eigene Volk gegen sich aufzuwiegeln. Hierbei nicht ins Straucheln zu geraten und abzustürzen ist sein Vermeidungsimperativ. So hangelt er sich von Woche zu Woche weiter, bis er dann doch aus dem Tritt gerät.

- Lebensführung unter Bedingungen hoher biographischer Ungewissheit heißt nicht nur, dass instruktive positive Zielsetzungen fehlen und allenfalls diffuse Vorhaben wie „in den nächsten Jahren promovieren“ ohne Verankerung in weiteren Lebensplänen im Raum stehen. Immer wieder sind sogar die Vermeidungsimperative nebulös. Man weiß nicht einmal, was man auf keinen Fall will oder tun sollte; man merkt es erst dann, wenn man den falschen Schritt bereits getan hat.

Der Scheitelpunkt von Zielausdünnung ist „Status quo als Argument“52: eine Wahrung dessen, was einen keineswegs begeistert und womit man oft sogar unzufrieden ist, ohne aber etwas greifbares Besseres vor Augen zu haben - doch man kann sich noch Schlechteres vorstellen. Wenn selbst diese Bestandserhaltung ohne 
Verbesserungsbestreben nicht mehr möglich ist, geht es nur noch darum, unabwendbare Verschlechterungen zu begrenzen, etwa zeitlich zu strecken.

Zielausdünnung ist die Schlussfolgerung daraus, dass entweder ein durchaus spezifizierbares Ziel auf absehbare Zeit völlig unerreichbar ist oder dass vorerst gänzlich unklar ist, was ein adäquates Ziel sein könnte. Weil es in beiden Fällen unmöglich erscheint, ex ante auch nur halbwegs "richtige“, also angemessene und realistisch erreichbare Ziele auszumachen, werden dafür erstens keine Energie und keine begrenzten Ressourcen verschwendet, sondern für bessere Verwendungen aufgespart; und zweitens werden Zieleingrenzungen aufgegeben, um so den Entscheidungshorizont zu öffnen. In diesen beiden Hinsichten ist Zielausdünnung durchaus noch begrenzt rational - besser als ein starres Festhalten an unerreichbaren oder illusionären Zielsetzungen.

Wenn der Entscheider über größere Strecken ratlos ist, was für inn längerfristig spezifizierbare Ziele sein könnten, kann er in diesen Zeiten nicht mehr tun als zu warten, bis sich das - wie und wodurch auch immer - klärt. Abwarten ist die zweite Komponente von Coping, die ebenfalls in allen drei betrachteten Fallbeispielen vorkommt:

- Für die kommissarische Behördenleiterin ist der durch Warten zu bewältigende Zeitraum relativ klar abgesteckt: Am nächsten Tag ist der stellvertretende Leiter von der Dienstreise zurück, und sie kann die Leitung an inn abgeben. Dessen größere Leitungserfahrung lässt inn möglicherweise routinierter mit Notfallsituationen $(\leftarrow$ p. 205) umgehen, sollte wieder eine solche eintreten. Die Behördenleiterin muss also nur einen Tag auf Zeit spielen.

- Der griechische Finanzminister musste sich über längere Zeiträume immer wieder vor allem aufs Hinhalten verlegen. Er setzte dabei insbesondere darauf, dass über Zeit auf allen Seiten Einsicht ins Notwendige wachsen würde: bei der griechischen Bevölkerung in die zu erbringenden großen Opfer und bei den ausländischen und internationalen Geldgebern darin, dass man Griechenland finanziell stärker und länger unter die Arme greifen muss, als man anfangs zu tun gewillt war.

- Biographisches Entscheiden unter Unsicherheit kennt ebenfalls längere Phasen des unentschlossenen Herumhängens, die man durchaus durchleidet, insbesondere wenn Außendruck auf endlich zu treffende Entscheidungen drängt. Hier ist darüber hinaus der Zeithorizont des Abwartens oftmals sehr unklar: Man 
kann nicht sagen, wie lange man noch brauchen wird, bis eine Entscheidung gereift ist und lässt gegebenenfalls eine Deadline nach der anderen - z.B. Einschreibtermine in einen Studiengang oder Wohnungsangebote - verstreichen, weil man das dumpfe Gefühl nicht los wird, dass es immer noch nicht „das Richtige" ist.

Es geht beim Abwarten wie für einen mittelmäßigen Flipperspieler um nicht mehr und nicht weniger als darum, den Ball im Spiel zu halten, um Zeit zu gewinnen - in der Hoffnung, dass sich dann ein "Lauf“ einstellt, in dem die Kugel ohne gezieltes eigenes Zutun punktet. ${ }^{53}$ Entscheidende können sich unter solchen Umständen als „Meister des Verzögerns, bis die Zeit reif ist“",) ${ }^{54}$ erweisen. Abwarten heißt freilich selten völliges Nichtstun - im Gegenteil: Der Flipperspieler ist ebenso wie der griechische Finanzminister und die Behördenleiterin sogar ziemlich hektisch beschäftigt. Doch was sie gleichermaßen zu vermeiden suchen, sind Entscheidungen, die den Status quo auf riskante Weise weitreichend verändern. Sie „verwalten“ - wie es unberechtigterweise abschätzig heißt - den deplorablen Status quo, weil sie entweder nicht wissen, wie ein besserer Zustand aussähe, oder keinen halbwegs sicheren Weg dorthin kennen.

Die begrenzte Rationalität dessen besteht zum einen genau darin: keine riskanten Experimente zu unternehmen, die unabsehbare - weitere - Verschlechterungen nach sich ziehen könnten. Zum anderen öffnet Abwarten den Zukunftshorizont dergestalt, dass aus dem mangelnden Wissen, was man tun sollte, der Schluss gezogen wird, Status-quo-Veränderungen auf eine unbestimmte Zeit des Ausschau-haltens zu vertagen. Die dahinter stehende Hoffnung eines „Kommt Zeit, kommt Rat!“ ist zumindest dann plausibel, wenn man nicht bei weiterem Nichtstun das schnelle Eintreten einer Katastrophe befürchten muss. ${ }^{55}$

Drittens ist in allen drei Fällen ein Zugreifen beobachtbar, sobald sich oftmals sehr unvorhergesehen und nur für kurze Zeit Gelegenheiten bieten, mehr als Abwarten $(\leftarrow$ p. 206) zu praktizieren. Zumeist läuft dem Entscheider dabei keine passfertige Option über den Weg, sondern er muss in Gestalt von „Basteln, Flicken, Probieren,

\footnotetext{
53 Schimank 1999.

54 Collingridge 1992, S. 151.

${ }^{55}$ Für Notfallmediziner oder Manager einer Naturkatastrophe ist Abwarten somit zumeist keine Option.
} 
Kombinieren"56 improvisieren - im Sinne eines reaktiven schnellen Nutzens von Gelegenheitsfenstern:

- Die Behördenleiterin kann vereinzelt Laufkunden an Mitarbeiter vermitteln, die unversehens etwas Zeit zwischen ihren Terminen haben. Dabei zählt freilich überhaupt nicht, wer schon wie lange gewartet hat, sondern allein, wer zufällig gerade vor ihr steht.

- Der griechische Finanzminister konnte bei mehreren Gelegenheiten nutzen, dass er durch Zufall bestimmte Gesprächspartner schon von früher kannte oder sie mehr Zeit für das Gespräch mit ihm hatten als vorgesehen, weil man z.B. länger auf dem Flughafen gemeinsam wartete. So konnte er hier und da für etwas mehr Verständnis für die griechische Situation werben. Auch eine unvorhergesehene bessere Entwicklung der griechischen Staatsfinanzen bot zu einem bestimmten Zeitpunkt die Chance, schneller mit der Haushaltskonsolidierung voranzukommen und so auch für Zufriedenheit der ausländischen Geldgeber zu sorgen. Eine andere "große Chance“ ließ die griechische Regierung hingegen, wie er rückblickend als Fehler konstatiert, aus: „anlässlich der Regional- und Kommunalwahlen kurzfristig parlamentarische Neuwahlen auszurufen und uns damit ein frisches Regierungsmandat zu holen. “57

- Biographisches Entscheiden muss, solange hohe Ungewissheit fortbesteht, auf teilweise lange Sequenzen des Improvisierens zurückgreifen. Wenn es gut geht, stellt es sich dann im Nachhinein so dar wie beim früheren Bundespräsidenten Roman Herzog: „Richard von Weizsäcker hat mich mal gefragt: ,Wie haben Sie Ihre Karriere geplant? Die ist so logisch aufgebaut.' Da habe ich gesagt: Wie ein Geißeltierchen (...). Es treibt im warmen Wasser, lässt die Fangarme spielen, und wenn was Interessantes vorbeikommt, schlägt es zu." ${ }^{38}$ So mancher muss freilich in unangenehm kaltem Wasser länger ausharren, ohne zu wissen, ob sich so bald was Besseres bieten wird.

Man kann also Gelegenheiten des Zugreifens erfolgreich nutzen; man kann sie aber auch verpassen, oder es kommen nie welche; und man kann vermeintliche Gelegenheiten nutzen, die sich dann als Fehler erweisen. Die Erfolgswahrscheinlichkeit ist dabei oftmals nicht gut abschätzbar, womit ein nicht unerhebliches Restrisiko bleibt. Die

\footnotetext{
56 Guttandin 1996, S. 31.

57 Papakonstantinou 2017, S. 211.

58 ZEIT-Magazin, 20.1.2011.
} 
begrenzte Rationalität des Zugreifens im Vergleich zum immer weiteren Warten ergibt sich aus folgendem Abwägen: Wie wahrscheinlich ist es, dass sich in absehbarer Zeit noch etwas Besseres bietet - und wie hoch ist umgekehrt das Risiko, jetzt einen die Situation deutlich verschlechternden Fehlgriff zu tun? Mit Blick auf letzteres sollte man sich zumindest vor weitreichenden irreversiblen Entscheidungen hüten.

Ob das, worauf zugegriffen wird, wirklich brauchbar ist, lässt sich ex ante nicht sagen. Es muss sich durch Ausprobieren erweisen. Eine vierte Komponente von $(\leftarrow$ p. 207) Coping ist dabei hilfreich: Sich-umfreuen. Es wird in den drei Fallbeispielen nicht explizit vorgeführt, kann aber durchaus plausibel unterstellt werden. Sich-umfreuen bedeutet: die Maßstäbe dafür, was eine „gute" Entscheidung ist, den Resultaten des Entscheidens anzupassen. Das kann zum einen ein Sich-arrangieren in Gestalt einer Anspruchsreduktion sein: Der Entscheider registriert, dass er einen zu großen Wirkungsgrad oder eine zu hohe Effizienz der Entscheidung erhofft hat, und macht Abstriche. Zum anderen kann der Entscheider auch feststellen, dass die Entscheidung zwar mit Blick auf die erwarteten Wirkungen wenig gebracht hat, dafür aber in ganz anderen Hinsichten durchaus positive Wirkungen zeitigt. Hierbei ergibt sich erst mit der Zeit aus den realisierten Gelegenheiten, deren improvisierender Nutzung und den sich einstellenden Resultaten, was man dann als erstrebenswerte Zielsetzungen mittlerer Reichweite ansieht. Diesbezüglich ist Coping - paradox formuliert - konsequent opportunistisch. ${ }^{59}$ Die Zielausdünnung des Entscheidens ermöglicht solch ein ex-post-Zurechtlegen zumeist bescheidener spezifischer Ziele als "schon immer so“ gewollt. Die Entscheiderin ist nahezu prinzipienlos lernbereit, wenn sie sieht, was an dem, was sie entschieden hat, zumindest einigermaßen funktioniert, so dass sie fürs Nächste sich und anderen sagen kann, was sie über ein bloßes Im-Spiel-Bleiben hinaus erreicht hat und weiter verfolgen will.

Auf die drei Fallbeispiele bezogen:

- Für die Behördenleiterin heißt Sich-umfreuen eine massive Anspruchsreduktion. Anstelle einer sachlich adäquaten, sozial gerechten und zügigen Bearbeitung der Anliegen der Kunden geht es nur noch darum, eine individuelle und noch mehr eine kollektive Rebellion der Kunden angesichts der offensichtlichen Unzulänglichkeiten und Ausfälle der organisationalen Leistungsproduktion zu

\footnotetext{
59 Luhmann 1975.
} 
vermeiden. Wenn zumindest das gelingt, kann die Behördenleiterin mit sich zufrieden sein.

- Beim griechischen Finanzminister wurde die anfängliche schockartige Einsicht, dass die neugewählte Regierung ihr ambitioniertes Reformprogramm vergessen konnte, weil sofort Schluss mit dem Schuldenmachen sein und stattdessen ein Schuldenabbau begonnen werden müsse, in der Folgezeit schnell ein von außen auferlegter, jedoch von ihm als grundsätzlich gerechtfertigt akzeptierter Zwang. Mit ersten kleinen Erfolgserlebnissen setzte dann ein Sich-umfreuen dahingehend ein, dass er damit zufrieden war, dass zum einen das Szenario eines anfangs sehr real drohenden Staatsbankrotts mitsamt Verlassen der Eurozone unwahrscheinlicher wurde und zum anderen der dafür implementierte Konsolidierungsprozess zeitlich gestreckt werden konnte, um Härten für die griechische Bevölkerung etwas zu mildern.

- Biographisches Entscheiden unter hoher Ungewissheit läuft, wie geschildert, auf die von Ronald Hitzler ${ }^{60}$ porträtierte „Bastelbiographie“ hinaus, bei der man sich immer wieder von sich selber, etwa von beruflichen Wendungen, überraschen lässt. Man könnte so etwas negativ als Enttäuschungen erleben: Beispielsweise hat es mit der Dissertation nicht geklappt, und daher musste anstelle der ersehnten $(\leftarrow$ p. 208) Wissenschaftlerlaufbahn ein „Brotjob“ angetreten werden. Oft genug findet aber auch ein Sinneswandel statt: Der „Brotjob“ fängt an, Spaß zu machen, weil er einen zwar nicht inhaltlich begeistert, aber man mit vielen netten Kollegen zusammenarbeitet, woraus schnell auch persönliche Freundschaften werden.

Begrenzt rational ist Sich-umfreuen, wenn man es als Haltung damit vergleicht, auf ewig dem, was unerreichbar ist, nachzutrauern oder ständig weiter vergeblich zu versuchen, im Nebel Ziele für sich auszumachen, die den Einsatz „wirklich“ wert sind. Beides führt zu nichts außer Zeitverschwendung und Frustration, die wiederum in dieser Gestalt völlig unproduktiv bleibt.

Diese vier Komponenten von Coping können, wie bisher dargestellt, in einem bestimmten Entscheidungsgeschehen je einzeln vorkommen, auch in Verbindung mit Komponenten der anderen beiden Entscheidungsmodi Planung und Inkrementalismus. Beispielsweise kann ein Planungsprozess durch äußere Umstände ins Stocken geraten, 
und dann kann Abwarten als Coping einsetzen; oder der Planungsprozess erzeugt völlig andere als die angestrebten Effekte, mit denen man sich jedoch durch Sich-umfreuen anfreunden kann. Ebenso könnte sich im Zuge einer inkrementalistischen „Politik der kleinen Schritte" plötzlich ein Gelegenheitsfenster auftun, das einem eine ungeahnte, aber sehr attraktive Option bietet; hier könnte der Inkrementalismus dann auf Coping als Zugreifen umschalten, um danach wieder weiter in kleinen Schritten - aber auf einem neuen Kurs - zu prozedieren. Umgekehrt kann aus Coping in den Modus der Planung oder des Inkrementalismus gewechselt werden, wenn eine entsprechend reduzierte Komplexität der Entscheidungssituation dies gestattet. Wenn sich etwa durch Sich-umfreuen der Zielhorizont des Akteurs radikal vereinfacht und in dieser Gestalt konsolidiert hat, kann auf einmal Planung oder zumindest Inkrementalismus in Gestalt von „satisficing“61 (sich mit dem Erstbesten zufrieden geben) möglich werden. In all diesen Fällen hat man es mit Hybridformen der Komplexitätsbewältigung zu tun, die entweder Komponenten des Coping in andere Entscheidungsmodi integrieren oder Elemente der anderen Modi in Coping. So wie es aber Planung und Inkrementalismus auch in Reinform gibt, existiert Coping in Reinform ebenfalls. Dann greifen die vier Komponenten systematisch ineinander, stehen also in einem sequentiellen Hervorbringungszusammenhang, der sich idealtypisch ausbuchstabiert so darstellt:

- Coping verlegt sich im ersten Schritt auf eine weitgehende Zielausdünnung.

- Als zweiter Coping-Schritt schließt sich daran Abwarten an: Wenn man nicht weiß, was man sinnvollerweise tun sollte, tut man erst einmal gar nichts.

In Orrin Klapps ${ }^{62}$ Konzeptualisierung von Komplexitätsbewältigung als Wechselspiel von „opening" und „closing“ stellt Zielausdünnung eine sachliche und soziale, Abwarten eine zeitliche Öffnung des Horizonts des weiteren Tuns dar. Darauf folgt eine Schließung dieses Horizonts zunächst in zeitlicher, sodann in sachlicher und sozialer Hinsicht: $(\leftarrow$ p. 209)

- Zugreifen als dritter Schritt, sobald sich eine Gelegenheit bietet, die mit einer gewissen Wahrscheinlichkeit Besseres verspricht als der Status quo,

- und Sich-umfreuen als Lernbereitschaft hinsichtlich dessen, was - wie unerwartet auch immer - funktioniert hat.

\footnotetext{
61 Simon 1976, S. 80-84.

621978.
} 
Wann immer Entscheider sich selbst Coping eingestehen, schwingt üblicherweise bedauernd mit, dass die Wichtigkeit des jeweiligen Problems eigentlich eine andere Umgangsweise erfordert hätte, die aber leider Gottes nicht möglich war. Vor dem Hintergrund des Planungsimperativs wird Coping schnell als defizitär abqualifiziert. Es kann deshalb zumeist auch nicht in Gestalt von rechtlichen oder organisationalen Verfahren sowie vorzeigbaren alltagsweltlichen Maximen „guten“ Entscheidens institutionalisiert werden, sondern bleibt ein eher uneingestandenes, wenngleich Kundigen wohlbekanntes und von ihnen bei Bedarf genutztes informelles Repertoire von Praktiken. Je wichtiger ein Problem ist, desto mehr gehören zum Coping daher auch Planungsfassaden in Richtung derjenigen, die den Entscheider argwöhnisch daraufhin beobachten, ob er planvoll agiert. Man tut dann so, als ob man einen gut überlegten Plan habe, damit z.B. Abwarten nicht als unverzeihliches Zaudern erscheint, sondern als schlaues Hinhalten.

\section{Ausblick}

Damit habe ich die Konturen von Coping anhand seiner vier Komponenten Zielausdünnung, Abwarten, Zugreifen und Sich-umfreuen - umrissen. Unter Hinzuziehung weiterer empirischer Fälle könnte man jede der Komponenten genauer als Repertoire von Praktiken differenzieren, also die Frage beantworten, auf welche verschiedenen Weisen Zielausdünnung, Abwarten, Zugreifen und Sich-umfreuen jeweils vollzogen werden können. Neben einer solchen genaueren Ausarbeitung und Prüfung des Konzepts sollte in der weiteren Diskussion aber vor allem auch geprüft werden, ob und inwieweit es einen zeitdiagnostischen Gehalt hat: Ist die Gegenwart und die voraussehbare Zukunft vielleicht zunehmend eine Gesellschaft des „Nur-noch-Coping"63?

Solch einer Hypothese stünde erst einmal entgegen, dass Coping überhaupt nichts Neues darstellt. So haben beispielsweise deutsche Kaiser des Hoch- und Spätmittelalters Praktiken des „dilatorischen Handelns“ gepflegt, also fällige Entscheidungen immer wieder hinausgeschoben und verzögert. Hintergrund war zumeist, dass sich die Herrscher in so diffizil gelagerten Kräfteverhältnissen bewegen mussten, dass jede Entscheidung in die eine oder andere Richtung unweigerlich Feindschaften erzeugt

63 Schimank 2011. 
hätte, die ein Herrscher sich nicht hätte leisten können. ${ }^{64}$ Francois Jullien ${ }^{65}$ zeigt Ähnliches für die klassische chinesische Kunstlehre der Diplomatie, der Politik und der Kriegsführung auf: „Warten können“, bis der richtige Moment gekommen ist. Man könnte noch viele weitere Fälle von Coping in vormodernen $(\leftarrow$ p. 210) gesellschaftlichen Verhältnissen oder auch in den 500 Jahren Moderne, die der gegenwärtigen Situation vorausgegangen sind, benennen und jeweils auf die äußerst komplexe Entscheidungssituation - und nicht bloß auf Planungsverweigerung aus Bequemlichkeit zurückführen.

Dennoch bleibt die Frage im Raum stehen, ob womöglich Coping heute zumindest häufiger und in mehr Gesellschaftsbereichen vorkommt als früher:

- Konkret könnte man für westliche Gesellschaften vermuten, dass nach dem Ende des gesellschaftlich sehr stabilen „Goldenen Zeitalter"66 von Anfang der 1950 er bis Mitte der 1970er Jahre die unruhigeren Zeiten, die seitdem angebrochen sind, zu einer Steigerung der Komplexität vieler Entscheidungssituationen von der individuellen Lebensführung bis zu Regierungshandeln geführt haben: ${ }^{67}$ Globalisierung, Ökonomisierung, Digitalisierung wären etwa Stichworte für in allen gesellschaftlichen Sphären wirkende Strukturdynamiken, die Treiber einer Komplexitätssteigerung sein könnten, wie sie sich als in sich widersprüchliche Mischung aus zunehmenden Ungewissheiten, Restriktionen und Knappheiten, einer Vervielfältigung von Möglichkeiten, Anspruchssteigerungen sowie einer gesellschaftlichen Beschleunigung manifestieren könnte. ${ }^{68}$

- Ergänzend oder alternativ zu einer solchen um sich greifenden gesellschaftlichen Komplexitätssteigerung könnte auch eingetreten sein, dass der Anspruch auf entscheidungsförmige Problembearbeitung auf immer mehr hochgradig komplexe Situationen ausgedehnt worden ist, die zuvor anders bewältigt, nicht zuletzt fatalistisch hingenommen worden sind. ${ }^{69}$ Solange u.a., wirksame Mittel zur Empfängnisverhütung nicht verfügbar waren, war Kinderkriegen nur in

\footnotetext{
64 Stollberg-Rillinger 2016, S. 19-34.

65 1996, S. 91-119.

66 Hobsbawm 1995, S. 324-362.

${ }^{67}$ Ariane Leendertz (2016) zeigt, dass seitdem in der gesellschaftlichen Selbstbeobachtung deutlich mehr von Komplexität gesprochen wird.

${ }^{68}$ Anstelle zahlreicher Studien, die hier angeführt werden könnten, verweise ich nur auf die - wenn auch stark auf Deutschland bezogene - Vieles ansprechende Überblicksdarstellung von Anselm Doering-Manteuffel und Lutz Raphael (2008).

${ }^{69}$ So für Individuen die bekannte These von Ulrich Beck 1986, S. 217.
} 
begrenztem Maße ein Gegenstand von Entscheiden; inzwischen gilt hierfür in vielen Milieus der Planungsimperativ, obwohl das Geschehen hochgradig unplanbar bleibt. ${ }^{70}$

Welche dieser beiden Diagnosen zutrifft, oder ob beide zugleich stimmen, kann hier zunächst dahingestellt bleiben, weil der Effekt derselbe ist: Sehr hohe Komplexität muss bzw. soll entscheidungsförmig bewältigt werden, was nur noch in Gestalt von Coping erfolgen kann.

Man sieht: Eine eingehende Beschäftigung mit Coping bringt nicht nur die soziologische Handlungstheorie und Entscheidungsforschung voran, sondern könnte auch eine wichtige Grundlage für ein besseres Verständnis der gegenwärtigen gesellschaftlichen Lage sein. $(\leftarrow$ p. 211)

\section{Literatur}

Beck, Hanno 2014, Behavioral Economics. Wiesbaden: Springer Gabler.

Beck, Ulrich 1986. Risikogesellschaft. Auf dem Weg in eine andere Moderne. Frankfurt/M: Suhrkamp.

Bonß, Wolfgang; Dimbath, Oliver; Hoffmeister, Helga; Maurer, Andrea; Nieder, Ludwig; Pelizäus-Schmid, Michael 2013. Handlungstheorie. Bielefeld: transcript.

Collingridge, David 1992. The Management of Scale: Big Organizations, Big Decisions, Big Mistakes. London: Routledge.

Danken, Thomas; Dribbisch, Katrin; Lange, Anne 2016. „Studying Wicked Problems Forty Years On: Towards a Synthesis of a Fragmented Debate", in der moderne staat 9, S. 15-33.

Doering-Manteuffel, Anselm; Lutz, Raphael 2008. Nach dem Boom. Perspektiven auf die Zeitgeschichte seit 1970. Göttingen: Vandenhoeck \& Ruprecht.

Dror, Yehezkel 1969 (1964). „Muddling Through - „Science“ or Inertia?”, in Readings on Modern Organizations, hrsg. v. Etzioni, Amitai, S. 166-171. Englewood Cliffs: Prentice Hall.

Esser, Hartmut 1990. „,Habits“, „Frames“ und Rational Choice. Die Reichweite der Theorie der rationalen Wahl (am Beispiel der Erklärung des Befragtenverhaltens)", in Zeitschrift für Soziologie 19, S. 231-247.

Esser, Hartmut 2001. Soziologie - Spezielle Grundlagen. Bd. 6: Sinn und Kultur. Frankfurt/M.: Campus.

Figueroa-Dreher, Silvana K. 2016. Improvisieren. Material, Interaktion, Haltung und Musik aus soziologischer Perspektive. Wiesbaden: Springer VS.

70 Heimerl, Hofmann 2016. 
Filipp, Sigrun-Heide; Aymanns, Peter 2010. Kritische Lebensereignisse und Lebenskrisen. Vom Umgang mit den Schattenseiten des Lebens. Stuttgart: Kohlhammer.

Guttandin, Friedhelm 1996. Improvisationsgesellschaft. Provinzstadtkultur in Südamerika. Pfaffenweiler: Centaurus.

Heimerl, Birgit; Hofmann, Peter 2016. „Wie konzipieren wir Kinderkriegen? Normativer Rationalismus versus empirische Praxisforschung“, in Zeitschrift für Soziologie 45, S. 410-430.

Hitzler, Ronald 1996. „Die Bastel-Existenz“, in Psychologie Heute 23, 7, S. 30-35.

Hobsbawn, Eric 1994 (1995). Das Zeitalter der Extreme. Weltgeschichte des 20. Jahrhunderts. München: Hanser.

Hodgkinson, Gerard P.; Starbuck, William H.. Hrsg. 2008. The Oxford Handbook of Orghanizational Decision-Making. Oxford: Oxford University Press.

Janis, Irving L.; Mann, Leon 1976. „Coping with Decisional Conflict”, in American Scientist 64, S. 657-667.

Janis, Irving L.; Mann, Leon 1977. Decision Making. A Psychological Analysis of Conflict, Choice, and Commitment. New York: Free Press.

Joas, Hans 1992. Die Kreativität des Handelns. Frankfurt/M.: Suhrkamp.

Joas, Hans; Knöbl, Wolfgang 2004. Sozialtheorie. Frankfurt/M.: Suhrkamp.

Jullien, Francois 1996 (1999). Über die Wirksamkeit. Berlin: Merve.

Kirsch, Werner 1977. Einführung in die Theorie der Entscheidungsprozesse. Wiesbaden: Gabler.

Klapp, Orrin E. 1978. Opening and Closing. Strategies of Information Adaptation in Society. Cambridge MA: Cambridge University Press.

Koppetsch, Cornelia 2013. Die Wiederkehr der Konformität. Streifzüge durch die gefährdete Mitte. Frankfurt/M.: Campus.

Kurt, Ronald; Näumann, Klaus. Hrsg. 2008. Menschliches Handeln als Improvisation. Sozial- und musikwissenschaftliche Perspektiven. Bielefeld: transcript.

Leendertz, Ariane 2016. „Das Komplexitätssyndrom. Gesellschaftliche „Komplexität“ als intellektuelle und politische Herausforderung in den 1970er Jahren", in Die neue Wirklichkeit. Semantische Neuvermessung und Politik seit den 1970er-Jahren, hrsg. v. Leendertz, Ariane; Meteling, Wenke, S. 93-132. Frankfurt/M.: Campus.

Lindblom, Charles E. 1959. „The Science of Muddling Through”, in Public Administration Review 13, S. 79-88.

Luhmann, Niklas 1964. „Lob der Routine“, in Politische Planung, S. 113-142. Opladen: Westdeutscher Verlag.

Luhmann, Niklas 1968. „Status quo als Argument“, in Studenten in Opposition, hrsg. v. Baier; Horst, S. 74-82. Bielefeld: Bertelsmann.

Luhmann, Niklas 1975 (1971). „Opportunismus und Programmatik in der öffentlichen Verwaltung“, in Politische Planung, S. 165-180. Opladen: Westdeutscher Verlag.

Meyer, John W. 2005. Weltkultur. Wie die westlichen Prinzipien die Welt durchdringen. Frankfurt/M.: Suhrkamp. 
Meyer, John W.; Jepperson, Ronald L. 2000. „The „Actors" of Modern Society: The Cultural Construction of Social Agency", in Sociological Theory 18, S. 100-120.

Papakonstantinou, Giorgios 2017. Game Over. Griechenland in der Krise: Der Insiderbericht. Wettingen: Kolchis.

Rittel, Horst; Webber, Melvin 1973. „Dilemmas in a General Theory of Planning”, in Policy Sciences 4, S. 155-169.

Schimank, Uwe 1999: „Flipperspielen und Lebenskunst“, in Identität und Moderne, hrsg. v. Willems, Herbert; Hahn, Alois, S. 250-272. Frankfurt/M.: Suhrkamp.

Schimank, Uwe 2005. Die Entscheidungsgesellschaft. Komplexität und Rationalität der Moderne. Wiesbaden: VS.

Schimank, Uwe 2009a. „Wichtigkeit, Komplexität und Rationalität von Entscheidungen", in Management komplexer Systeme - Konzepte für die Bewältigung von Intransparenz, Unsicherheit und Chaos, hrsg. v. Weyer, Johannes; Schulz-Schaeffer, Ingo, S. 55-71. München: Oldenbourg.

Schimank, Uwe 2009b: Die „reflexive Moderne“: eine wohlbekannte Entscheidungsgesellschaft, in Handeln unter Unsicherheit, hrsg. v. Böhle, Fritz; Weihrich, Margit, S. 7793. Wiesbaden: VS.

Schimank, Uwe 2011. „Nur noch Coping: Eine Skizze postheroischer Politik“, in Zeitschrift für Politikwissenschaft 21, S. 455-463.

Schimank, Uwe 2015. „Lebensplanung!? Biografische Entscheidungspraktiken irritierter Mittelschichten“, in Berliner Journal für Soziologie 25, S. 7-31.

Schmidt, Robert 2018. „Entscheiden als retroaktives Regelfolgen“, in Kulturen des Entscheidens. Narrative - Praktiken - Ressourcen, hrsg. v. Pfister, Ulrich, S. 52-67. Göttingen: Vandenhoeck \& Ruprecht.

Schubert, Hans-Joachim 2009. „Pragmatismus und Symbolischer Interaktionismus“, in Handbuch Soziologische Theorien, hrsg. v. Kneer, Georg; Schroer, Markus, S. 345367. Wiesbaden: VS.

Simon, Herbert A. 1976 (1946). Administrative Behavior. A Study of Decision-Making Processes in Administrative Organization. New York: Free Press.

Stoll, Florian 2012. Leben im Moment? Soziale Milieus in Brasilien und ihr Umgang mit Zeit. Frankfurt/M.: Campus.

Stollberg-Rilinger, Barbara 2016. Cultures of Decision-Making. London: German Historical Institute London. 


\section{Zusammenfassung}

Wie können Entscheidungshandelnde mit sehr komplexen Problemsituationen zurechtkommen, die ein geordnetes Abwägen von Alternativen unmöglich machen? Der Beitrag stellt "Coping“ als einen in solchen Situationen zum Zuge kommenden Modus des Entscheidens dar. Als empirische Anker der Argumentation dienen drei Fallbeispiele individuellen, organisationalen und politischen Entscheidens.

\section{Summary}

How can decision-makers handle very complex problem situations in which a careful consideration of alternatives becomes impossible? The article presents "coping" as a mode of decision-making which is used in such situations. Three case studies of individual, organisational, and political decision-making serve as empirical illustrations of the argument.

\section{Stichworte}

Entscheiden - Komplexität - "wicked problems" - Rationalität - Planung

\section{Keywords}

Decision-making - complexity - "wicked problems" - rationality - planning

\section{Englischer Titel:}

\section{Coping - Decision-making Where It is Nearly Impossible}

\section{Autorenangaben:}

Prof. Dr. Uwe Schimank

Universität Bremen

SOCIUM - Forschungszentrum Ungleichheit und Sozialpolitik

Mary-Somerville-Str. 9

\section{Bremen}

Uwe.schimank@uni-bremen.de 